\title{
EVALUACIÓN DE LA VIDA ÚTIL DE LOS ALIMENTOS (SHELF LIFE): EFECTO DE LA TEMPERATURA
}

Elí J. Espinoza Atencia ${ }^{1}$

\author{
RES U M E N
}

\begin{abstract}
La vida títil de los alimentos depende del nivel tecnologico utilizado en el procesamiento y del sistema envase-alimento-medio ambiente. Dentro de los factores ambientales, la temperatura es el más importante durante el almacenamiento y distribución de alimentos. En esta publicación se tratan aspectos que relacionan la temperatura y la cinética del deterioro de los alimentos mediante ecuaciones matemaiticas.
\end{abstract}

\section{A B STRACT}

The shelf life of food depends on the technological level used in the processing as well as on the food packaging-environment system. Within the environmental factors, the temperature is the most important to be considered during the storage and distribution of food. This publication deals with aspects that relate the temperature and the kinetics of deterioration of food through mathematical equations.

\section{INTRODUCCIÓN}

Es evidente el interés actual sobre el deterioro que sufren los alimentos en forma inevitable, durante los procesos de elaboración, envasado y distribución. Este interés es debido en gran parte a las exigencias que las autoridades sanitarias imponen con el marcado de fechas de consumo en las etiquetas de estos alimentos, principalmente en los países con legislación alimentaria más avanzada.

Dada la complejidad de los productos alimenticios, puede afirmarse que existen pocos estudjos en profundidad que aborden la cuantificación del periodo de consumo de los alimentos, sólo últimamente y debido al avance en el conocimiento cinético de los diversos procesos de deterioro, sus fenómenos y agentes, asi como el uso de métodos analíticos y el auxilio de la informática, que permite la simulación del comportamiento de sistemas complejos, se están efectuando avances prometedores.

1. Doctor en Ingeniería de Alimentos
En esencia, los procedimientos para la determinación de una fecha de consumo preferente, para asemejarla al "shelf life" anglosajón, se puede denominar vida comercial o vida útil del alimento, pasan por el establecimiento de un modelo matemático, con una serie de ecuaciones que simulan el deterioro que sufren los alimentos en diversas situaciones ambientales. De esta manera, pueden hallarse alternativas con las que se mejore su procesado, almacenaje y ampliar su vida útil y quizás sea más interesante, predecir esta vida útil, en nuevos productos y alimentos ya tradicionales, que por técnicas deba efectuarse alguna variación en su composición o proceso.

En esta serie de trabajos a publicarse, primero se presenta una revisión de los agentes más importantes que influyen en el deterioro de los alimentos, para luego dar algunas pautas que permitan construir un modelo cinético-matemático de este deterioro. Finalmente, aplicar la teoría a los diferentes casos especificos. 


\section{FACTORES QUE AFECTAN EL DETERIORO DE LOS ALIMENTOS}

Si se quiere establecer un modelo cinéticomatemático del deterioro de un alimento, el primer paso será conocer a fondo los factores que influyen y la forma de su acción. La aproximación cinética debe basarse en la velocidad de este proceso de deterioro, el cual está relacionado a factores ambientales (temperatura, presión, humedad) y factores de composición (concentración, $\mathrm{pH}$, etc.) (SUGUY \& KAREL, 1980). La ecuación que describe esta pérdida de calidad está dada por:

$$
\mathrm{dC} / \mathrm{dt}=f(\mathrm{Ei}, \mathrm{Fj})
$$

donde:

$$
\begin{aligned}
\mathrm{dC} / \mathrm{dt} & =\text { Velocidad de deterioro } \\
\mathrm{Ei} & =\text { Factores ambientales }(i=1,2, \ldots \mathrm{n}) \\
\mathrm{Fj} & =\text { Factores de composición }(j=1,2, \ldots \mathrm{m})
\end{aligned}
$$

Los efectos de algunos de estos factores se pueden expresar con relativa facilidad en una relación funcional que es similar en muchas reacciones de deterioro, otros son más complicados y únicos en su comportamiento y deberán derivarse por separado para cada producto y sistema alimenticio.

Existen relaciones funcionales generales para la temperatura, concentración, actividad de agua y contenido acuoso, oxígeno y modelos mixtos, en que intervienen más de uno de estos factores, los cuales comentaremos por separado.

\subsection{EFECTO DE LA TEMPERATURA}

Este es el factor del ambiente que más afecta la conservación de los alimentos durante el almacenamiento y comercialización. Todas las alteraciones que sean de naturaleza biológica o físicoquimica presentan tasas de transformaciones que varian con la temperatura del ambiente.

Existen varias maneras de expresar matemáticamente, la relación existente entre la temperatura y la velocidad de deterioro de un alimento. Por ejemplo, se tiene la expresión $Q_{10}$ definida por la ecuación (2) y (3).

$$
\begin{aligned}
& Q_{10}=\frac{\text { Velocidad de reacción }(T+10)}{\text { Velocidad de reacción } T} \\
& Q_{10}=\frac{\text { Vida útil }(T+10)}{\text { Vida útil } T}=\frac{t s(T)}{t s(T+10)}
\end{aligned}
$$

donde:

$$
\begin{aligned}
& \mathrm{T}=\text { Temperatura }\left({ }^{\circ} \mathrm{C}\right) \\
& \text { ts = Vida útil }
\end{aligned}
$$

El $Q_{10}$ representa la razón obtenida por el aumento de la velocidad de las reacciones cuando la temperatura del sistema aumenta en $10^{\circ} \mathrm{C}$. Para reacciones enzimáticas un $Q_{10}=2$ es frecuentemente encontrado. Esto indica que la velocidad enzimática se dobla para cada $10^{\circ} \mathrm{C}$ de aumento en la temperatura, hasta que ocurra la desnaturalización o inactividad de la enzima por la energía calorífica (LABUZA \& RIBOH, 1982).

De acuerdo a la Ley de Van't Hoff, el aumento de la velocidad de la reacción, para una diferencia de temperatura $\square$, diferente a $10^{\circ} \mathrm{C}$, se cumple la relación:

$$
\left[Q_{10}\right]^{\square / 10}=\frac{t_{(\mathrm{T} 1)}}{t_{(\mathrm{T} 2)}}
$$

donde:

$$
\begin{aligned}
\square & =T_{2}-T_{1} \\
\text { ts }_{(T 1)} & =\text { Tiempo de vida útil a la temperatura } T_{1} \\
t_{(T 2)} & =\text { Tiempo de vida útil a la temperatura } T_{2}
\end{aligned}
$$

Por ejemplo, si el Q10 es 3 y la vida útil fue 6 meses a $35^{\circ} \mathrm{C}$, la vida útil a $20^{\circ} \mathrm{C}$ podrá ser :

$$
t_{20}=t_{35} \cdot Q_{10}^{\square 10}=6 \cdot 3^{15 / 10}=31,2 \text { meses }
$$

Por ejemplo, si la vida útil de un producto fue de dos semanas a $50^{\circ} \mathrm{C}$, la Tabla $\mathrm{N}^{\circ} 1$ muestra que la predicción de la vida útil podría ser realizada a diferentes temperaturas y varios valores de $Q_{10}$, asumiendo que los valores de $Q_{10}$ son constantes, una diferencia de 0,5 en el valor de $Q_{10}$ puede tener un gran efecto en la predicción de la vida útil. (LABUZA \& RIBOH, 1982)

TABLA N ${ }^{\circ}$ 1: Efectos de la temperatura en la vida útil.

\begin{tabular}{|c|c|c|c|c|}
\hline \multirow{2}{*}{$\begin{array}{c}\text { TEMPERATURA } \\
\left({ }^{\circ} \mathrm{C}\right)\end{array}$} & \multicolumn{5}{|c|}{ VIDA ÚTIL (semanas) } \\
\cline { 2 - 5 } & $\mathrm{Q}_{10}=2$ & $\mathrm{Q}_{10}=2,5$ & $\mathrm{Q}_{10}=3$ & $\mathrm{Q}_{10}=5$ \\
\hline 50 & 2 & 2 & 3 & 2 \\
40 & 4 & 5 & 6 & 10 \\
30 & 8 & 12,5 & 18 & 50 \\
20 & 16 & 31,3 & 54 & - \\
\hline
\end{tabular}

FUENTE: LABUZA \& RIBOH (1982) 
El $Q_{10}$ es una constante $\sim$ 2, pero puede variar en función de una gran variación de temperatura (Tabla $N^{\circ} 2$ ). Por ejemplo, las enzimas que gobiernan las reacciones bioquimicas, pueden ser afectadas por la temperatura. En general, las actividades enzimáticas en frutos de hortalizas decae a temperaturas superiores a $30^{\circ} \mathrm{C}$, algunas enzimas son activas a $35^{\circ} \mathrm{C}$, pero la mayoría es inactivada a $40^{\circ} \mathrm{C}$. A, temperaturas superiores a $35^{\circ} \mathrm{C}$ se torna anormal, lo que resulta en rompimiento de la integridad y estructura de las membranas celulares (KADER, 1985).

TABLA N ${ }^{\circ}$ 2: Efecto de la temperatura sobre la tasa de deterioración de productos no sensibles al frío.

\begin{tabular}{|c|c|c|c|c|}
\hline $\begin{array}{c}\text { TEMPERATURA } \\
\left({ }^{\circ} \mathrm{C}\right)\end{array}$ & $\begin{array}{c}\mathbf{Q}_{10} \\
\text { ASUMIDO }\end{array}$ & $\begin{array}{c}\text { VELOCIDAD } \\
\text { RELATIVA DE } \\
\text { DETERIORAC. }\end{array}$ & $\begin{array}{l}\text { VIDA ÚTIL } \\
\text { RELATIVA }\end{array}$ & $\begin{array}{c}\text { PÉRDIDA } \\
\text { DIARIA } \\
(\%)\end{array}$ \\
\hline 0 & - & 1,0 & 100 & 1 \\
10 & 3,5 & 3,0 & 33 & 3 \\
20 & 2,5 & 7,5 & 13 & 8 \\
30 & 2,0 & 17,0 & 7 & 14 \\
40 & 1,5 & 22,5 & 4 & 25 \\
\hline
\end{tabular}

FUENTE: KADER (1985)

La manera más clásica de representar la velocidad de deterioro o de reacción en función de la temperatura, es por medio de la ecuación de Arrhenius:

$$
K=K_{0} e^{-E_{A} / R T}
$$

donde:

$\mathrm{K}=\mathrm{Es}$ la constante de velocidad de deterioro.

$\mathrm{K}_{\mathrm{O}}=$ Factor de frecuencia, también denominada preexponencial (constante independiente de la temperatura).

$\mathrm{E}_{\mathrm{A}}=$ Energía de activación en $\mathrm{kcal} / \mathrm{mol}$. $\mathrm{K}$

$\mathrm{R}=$ Constante universal de los gases en kcal/ mol;

$\mathrm{T}=$ Temperatura absoluta en $\mathrm{K}$.

La forma logaritmica de la ecuación (5) es la siguiente:

$$
\log K=-E_{A} / 2,3 R T+\log K_{0}
$$

La energía de activación $\left(E_{A}\right)$ se puede deducir de un gráfico logarítmico, que representa Log K versus $1 / T$, si se obtiene una linea recta, sigue la ecuación de Arrhenius. BONE (1973) encontró una relación lineal entre Log de la velocidad de la reacción o deterioro $(\log K)$ y la inversa de la temperatura absoluta (1/T). El coeficiente angular de la recta está relacionado con la energía de activación como sigue:

$$
\text { Coeficiente angular }=E_{A} / 2,3 R
$$

La inclinación de la línea trazada representa $E_{A} / R$ asi se puede obtener la energía de activación (Fig. $N^{\circ} 1$ ). La energía de activación depende de las características asociadas a la composición de cada alimento como puede ser su actividad de agua (Aw), concentración de sólidos, $\mathrm{pH}$ y otros.

Es preferible determinar el $Q_{10}$ a partir de la energia de activación conforme a la siguiente ecuación (8):

$$
\log Q_{10}=2,2 E_{A} / T(T+10)
$$

El uso de la $E_{A}$ y del Q10 permite una mejor aplicación racional del tratamiento térmico y del manejo de las temperaturas de almacenamiento. En productos almacenados, una reducción de $10^{\circ} \mathrm{C}$ en la temperatura de almacenamiento, puede duplicar la vida útil de los mismos. Pero es necesario tener conocimiento del mecanismo de las deterioraciones, de modo de no hacer estimados incorrectos, considerando que la energia de activación puede variar cuando se investiga un gran intervalo de temperaturas. Tal fenómeno es común en reacciones oxidativas a altas temperaturas, mayores de $100^{\circ} \mathrm{C}$.

FIGURA N ${ }^{\circ}$ 1: Representación esquemática de la ecuación de Arrhenius.

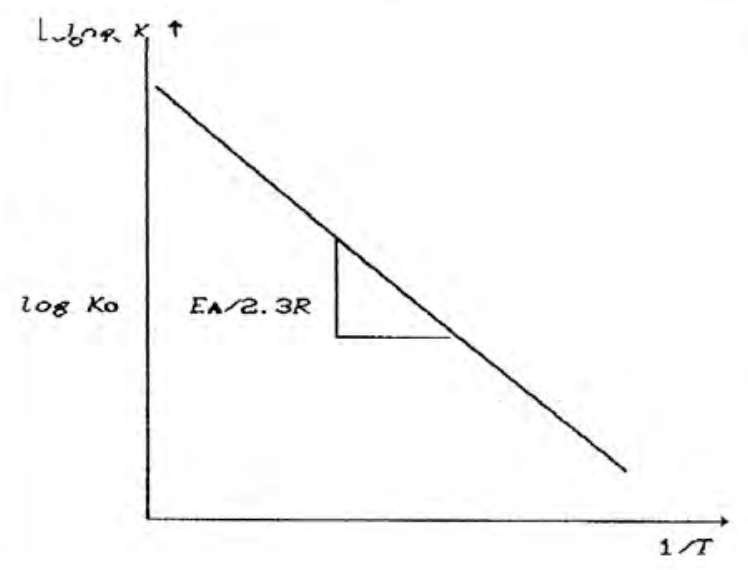


El uso del valor $Z$, es otra forma de representar el efecto de la temperatura en la velocidad de las transformaciones de los alimentos. El valor Z, representa el intervalo de temperatura que ocasiona una variación de 10 veces en la velocidad de transformación. Para la obtención del valor $Z$ de una reacción, generalmente se coloca en un gráfico semilogarítmico el tiempo necesario para que una reacción llegue a un determinado limite, o también el valor $D$ definido versus la temperatura, en la reacción en que se verifica. (Fig. $\mathrm{N}^{\circ} 2$ )

El comportamiento de las reacciones a diferentes temperaturas es mostrado en gráficos en escala lineal (Fig. $\mathrm{N}^{\circ} 3$ ) y semilogaritmico (Fig. $\mathrm{N}^{\circ} 4$ ), asi el valor de $Q_{10}$ puede ser calculado.

Como fue mencionado $E_{A}, Z$ y $Q_{10}$ se expresan de diferentes formas, como la velocidad de una transformación depende de la temperatura. Así existen relaciones matemáticas entre estos valores y están representados por la ecuación (9):

$$
Z=\frac{4,6 T^{2}}{E_{A}}
$$

donde:

$$
\begin{aligned}
& T=\text { Temperatura en } \mathrm{K} \\
& E_{A}=\text { Energía de activación cal } / \mathrm{g} \mathrm{mol} \\
& Z={ }^{\circ} \mathrm{C}
\end{aligned}
$$

La relación entre $\mathrm{C}$ y el valor de Z es la siguiente:

$$
Z=\frac{10}{\log Q_{10}}
$$

Finalmente relacionando las ecuaciones (9) y (10) se tiene:

$$
E_{A}=0,46 T^{2} \log Q_{10}
$$

donde:

$$
\begin{aligned}
& \mathrm{T}=\text { temperatura en } \mathrm{K} \\
& \mathrm{Q}_{10}={ }^{\circ} \mathrm{C}
\end{aligned}
$$

Por definición $Q_{10}$ es expresado en ${ }^{\circ} \mathrm{C}$, pero alguna literatura especializada expresa en ${ }^{\circ} \mathrm{F}$. En este caso existe la relación:

$$
\begin{gathered}
\log \left(Q_{10}{ }^{\circ} \mathrm{C}\right) / \log \left(Q_{10}{ }^{\circ} \mathrm{F}\right)=1,8 \\
\left(\mathrm{Z} \text { en }{ }^{\circ} \mathrm{F}\right)=\left(\mathrm{Z} \text { en }{ }^{\circ} \mathrm{C}\right) \cdot 1,8
\end{gathered}
$$

FIGURA N ${ }^{\circ}$ 2: Efecto de la temperatura de almacenamiento en la vida útil. Carne de pollo refrigerado. (VAN ARSDEL, 1969)

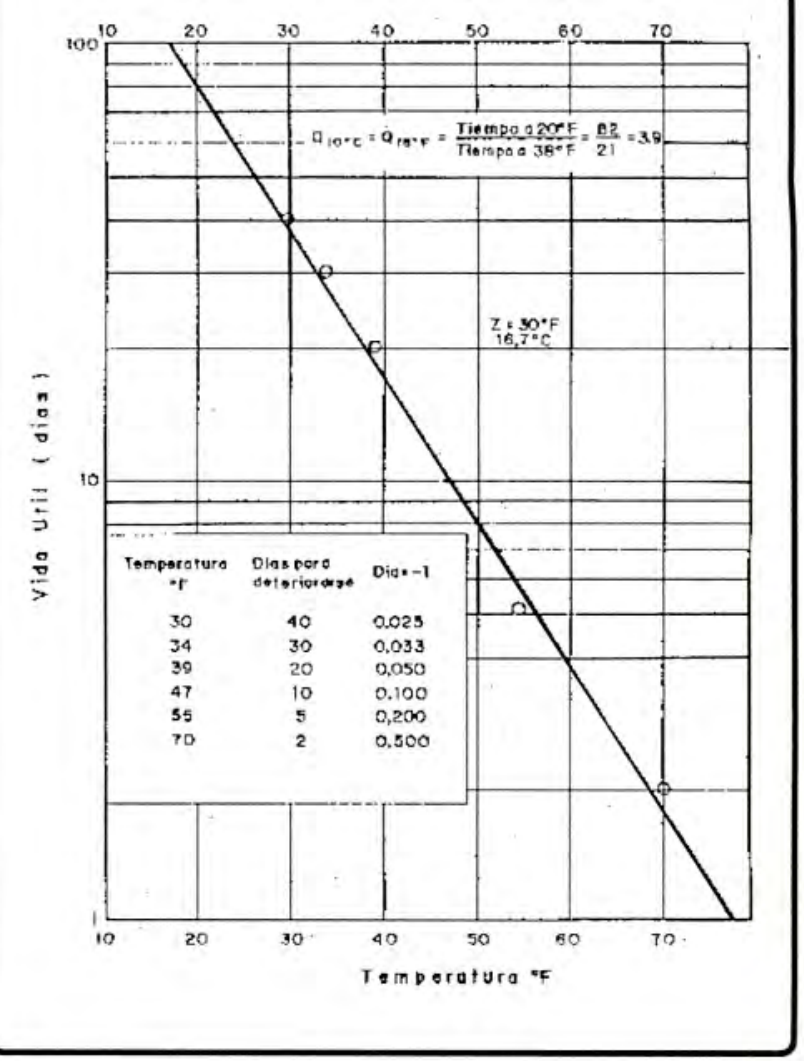

FIGURA $N^{\circ}$ 3: Destrucción de la vitamina $\mathrm{C}$ en un alimento deshidratado (gráfico lineal).

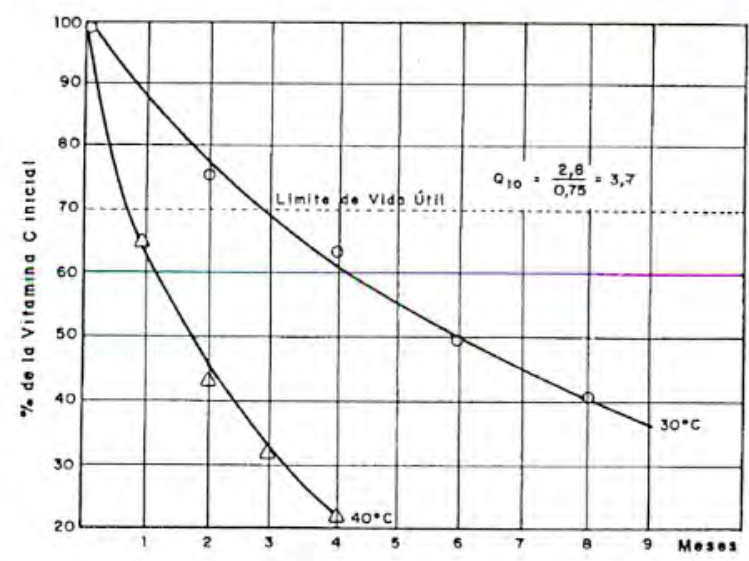


FIGURA N ${ }^{\circ}$ 4: Destrucción de la vitamina $C$ en un alimento deshidratado (gráfico semilogarítmico).

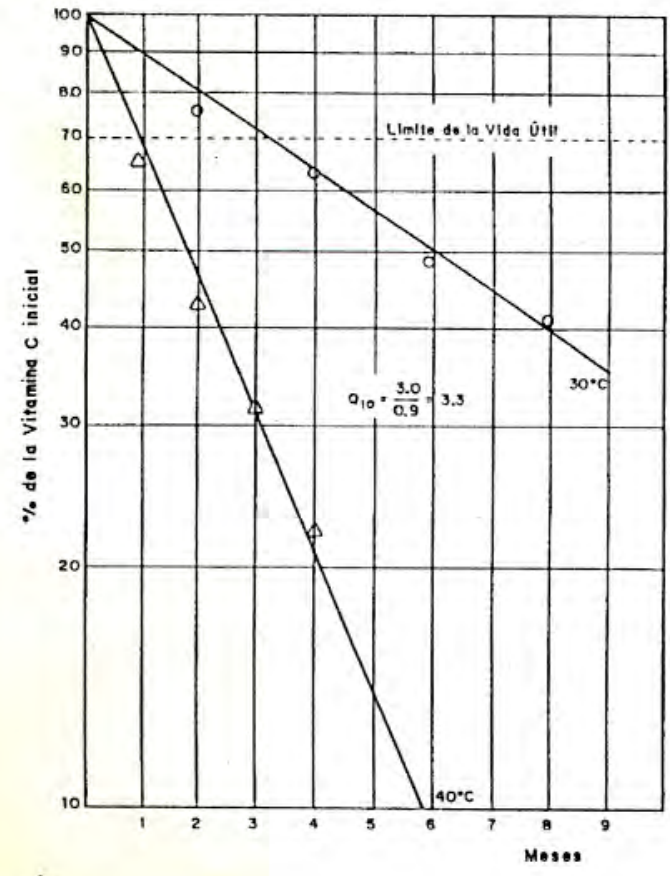

Es importante mencionar que cuanto mayor fuera el valor de $Z$, menor será la dependencia de la transformación en relación a la temperatura. De la misma forma, cuanto mayor fuera el valor de $Q_{10} y / o$ $\mathrm{E}_{\mathrm{A}}$, mayor será la dependencia de la transformación en

\section{BIBLIOGRAFÍA}

BONE, D. ; Water activity in intermediate moisture foods. Food Technology 27(4): 71-76, 1973.

CABRAL, A.C. \& FERNANDES, M.H.; Aspectos gerais sobre a vida de prateleira de produtos alimenticios. Bol ITAL, campinas 17(4): $371-439,1980$

FARIA, J.A.F.; Estabilidade de Alimentos em Embalagens Plásticas Universidade estadual de campinas. UNICAMP/FEA, 1990.

INSTITUTE OF FOOD TECHNOLOGGISTS (IFT); Shelf life of food technology. Food Technology 28(8):44-47, 1974.

KADER, A.; An overview of the physiological and biochemical basis of Cla effects on fresh horticultural crops. Proceedings of Fourth National Controlled Atmosphere Research Conference University of California, July, 1985

KAREL, M.; Effects of packaging in maintenance of nutrients in food products. In: HARRIS R.S. \& VON LOESECKE, H.: Nutritional of Foods Processing. Westport. Avi. p. 305-336, 1971.

KAREL, M.; Recent research and development in the field lowmoisture and intermediate-moisture foods. Critical Reviews in Food Technology 3(4): 329-373, 1973.

KAREL, M.; Water activity and food preservation in principles of food science In: FENNEMA, O.R.; Physical Principles of Food Preservation. Marcel Dekker Inc., New York, p: 237-263, 1975. relación a la temperatura. Todo esto comparado a un mismo intervalo de temperatura (TEIXEIRA et alii, 1991). En la Tabla $N^{\circ} 3$, se presentan valores $Z, E_{A}$ y $D$ para algunos constituyentes de los alimentos.

Conociendo la energía de activación para cierto rango de temperatura y la constante de reacción $\left(\mathrm{K}_{1}\right)$ a una temperatura dada $\left(T_{1}\right)$, se puede calcular la constante $\left(\mathrm{K}_{2}\right)$ para la temperatura $\left(\mathrm{T}_{2}\right)$ conforme a la ecuación (LABUZA, 1968). Este cálculo es más correcto que los determinados en base a $Q_{10}$, pues éste varía en función de la temperatura o mejor en función de la energía de activación.

$$
\text { In } K_{2} / K_{1}=-E_{A} / R\left(1 / T_{2}-1 / T_{1}\right)
$$

Las unidades de energía de activación $\left(E_{A}\right)$ y la constante de los gases $(R)$ deben ser compatibles, tales como $\mathrm{kcal} / \mathrm{mol}$. K.

TABLA N ${ }^{\circ}$ 3: Resistencia térmica de los constituyentes alimenticios.

\begin{tabular}{|l|c|c|c|}
\hline \multicolumn{1}{|c|}{ CONSTITUYENTES } & $\mathrm{Z}$ & $\mathrm{E}_{\mathrm{A}}$ & $\mathrm{D}_{121}$ \\
\hline VITAMINA & $44-55$ & $20-30$ & $100-1000$ \\
COLOR, TEXTURA, $\mathrm{Y}$ & & & \\
SABOR & $45-80$ & $10-30$ & $5-500$ \\
ENZIMAS & $12-100$ & $12-100$ & $1-10$ \\
CÉLULAS VEGETALES & $8-12$ & $100-120$ & $0,002-0,02$ \\
ESPORAS & $12-22$ & $53-83$ & $0,1-5,0$ \\
\hline
\end{tabular}

FUENTE: LUND(1979)

KAREL, M. \& HEIDELBAUGH, N.D.; Efects of packaging on nutrients. In HARRIS, R.S. \& KARMAS, E.; Nutritional of Foods Processing Westport. AVI. p. 412, 1975.

KRAMER, A.; Storage retention of nutrints. Food Technology. 28(1): 50 $60,1974$.

LABUZA, T.P.; Sorption phenomena in foods. Food Technology, Chicago, 22(3): 15-24, 1968.

LABUZA,T,F ; Aplication of chemical kinetics to deterioration of foods J. Chem. Educ, 61: 348, 1984.

LUNDBERG,W.O.; Mechanisms in: SCHULTZ.H.W. DAYE.A \& SINNHUBER, R.O.; Lipids their oxidation. Westport. AVI, p: 31 50. 1962

PASSY, N. \& MANNHEIM, C.; Vida-de-prateleira para alimentos. In: I Congresso Brasileiro de Enbalagem, São Paulo, Marco, 1977

SALWIN, H. \& SLWSON, V: Moisture in combinations of dehidrated foods Food Technology 13(12):715-718, 1959.

TEXEIRA, R.O: Reacōes de Transformacão e vida-prateleira de Alimentos procesados. Manual Técnico $\mathrm{N}^{\circ} 6$, Instituto de Tecnología de Alimentos (ITAL), Sāo Paulo, Brasil, 1991

VAN ARSDEL, W.B.; COPLEY, M.J. \& OLSON. R.L.; Quality and stability of Frozen Foods. Willey Interscience, New York, 1969 